\title{
Jitter performance for QoS in Ethernet Passive Optical Networks
}

\author{
Abhishek Dixit, Goutam Das, Bart Lannoo, Didier Colle, Mario Pickavet, Piet Demeester \\ Department of Information Technology, Ghent University - IBBT, B-9050 Gent, Belgium \\ abhishek.dixit@intec.ugent.be
}

\begin{abstract}
We propose a new online dynamic bandwidth allocation algorithm for QoS in EPON, which provides a constant delay and jitter performance to higher and medium priority traffic classes while maintaining a high throughput.

OCIS codes: (060.4250) Networks; (060.4252) Networks, broadcast
\end{abstract}

\section{Introduction}

Emerging applications like voice-over-IP (VOIP), IPTV, broadband data, and peer to peer sharing require a guaranteed bound on many parameters like: bandwidth, packet delay (latency), delay variation (jitter), and packetloss ratio. Quality of Service (QoS) refers to a network's ability to provide bounds on some or all of the above parameters. The paper focuses on QoS issues in EPON networks.

To achieve an effective resource allocation in EPONs, several schemes using dynamic bandwidth allocation algorithms (DBA) have been proposed, such as Interleaved Polling with Adaptive Cycle Time (IPACT) [1]. IPACT achieves high throughput but the variable cycle time of the algorithm is not suitable for the high priority jitter sensitive applications [2,3]. To provide constant jitter performance, several fixed cycle time scheduling algorithms (such as Hybrid Slot-Size/Rate protocol (HSSR) [4], Cyclic-Polling-based Bandwidth Allocation with SLAs (CPBA-SLA) [3]) or approaches with separate cycle for each traffic class (such as Hybrid Grant Protocol (HGP) protocol [2]) have been proposed. The fixed frame (cycle time) algorithms limit the channel utilization in the context of highly bursty traffic. In HGP protocol, there is a separate cycle for each traffic class and there are idle periods between each cycle which limits the throughput. Thus, there is a need for an algorithm which provides constant delay variation to high priority traffic with high throughput. Also, with emergence of many applications (like interactive or streaming video); a service differentiation is needed for even medium priority traffic. An algorithm must be able to serve applications according to the specified parameter bound (leading to the parameterized QoS control).

We propose the Delay-Aware Window Sizing (DAWS) approach (based on IPACT) to reduce the delay and the delay variation for high priority traffic. We achieve an improved jitter performance and a considerable higher throughput. For medium priority traffic, we propose the Delay-Aware Grant Sizing (DAGS) approach which helps to maintain the average delay according to the specified parameter and minimizes jitter. The simulation results show the effectiveness of our proposed algorithm. The rest of the paper is organized as follows. Section 2 presents our new protocol. Detailed performance analysis is done in Section 3. Section 4 concludes the paper.

\section{Algorithm}

Based on the required bound, we categorize the traffic into three different classes: a) EF (Expedited Forwarding) highest priority, delay sensitive traffic with constant bit rate such as voice transmission, b) AF (Assured Forwarding) - medium priority, delay sensitive traffic with variable bit rate such as video transmission and c) $B E$ (Best Effort) - low priority traffic for non-real time data transfer such as e-mail applications. We will now discuss how low delay and jitter bounds are met for the EF and AF traffic class.

DAWS (for EF traffic): For non-bursty EF traffic, the delay variation of the first departed EF packet between two consecutive transmission windows (inter-window jitter), maps the distribution property of the total EF delay sequence of an ONU [2]. EF packets with more fluctuation in their inter-window jitter tend to be over delayed or under delayed with respect to their mean value, and so the total EF delay sequence appears to be more dispersed. The inter-window jitter between the $i^{\text {th }}$ window and the $(i+1)^{\text {th }}$ window $J_{i}$ is given by (1), where $D_{i}$ is the delay of the first departed packet within the $i^{\text {th }}$ window [3].

$$
J_{i}=D_{i}-D_{i+1}
$$

For predictable EF traffic, the grant-before-request (GBR) method has been suggested in [2]. In the GBR method, the EF traffic will be granted before a request is made from an ONU and the maximum delay bound of the 
EF traffic $\left(D_{\mathrm{EF}}\right)$ is equal to the maximum cycle time. Fig. 1(a) illustrates when the GBR method is combined with IPACT, resulting in a delay variation of the first departed EF packet. We can see that the delay of the first departed EF packet (represented by $\mathrm{D}_{\mathrm{i}}, \mathrm{D}_{\mathrm{i}+1}, \mathrm{D}_{\mathrm{i}+2}$ ) is nearly equal to the cycle time. As the cycle time is variable, the EF packets will experience variable delay. In our DAWS algorithm, we adopt delay-aware GBR approach, and the OLT allocate the transmission slot for an ONU such that the delay of the first departed EF packet at the considered ONU remains constant. The OLT delays the transmission of some packets (by granting a smaller transmission slot) to the subsequent cycle, if even doing so, does not increase the delay of the packets beyond $\mathrm{D}_{\mathrm{EF}}$. If $\mathrm{w}[\mathrm{p}]$ is the transmission slot for an ONU p, $\mathrm{x}$ is the time duration of the present cycle, $\mathrm{y}$ is the expected time duration of the next cycle, then the maximum delay for packets ungranted in the present cycle is $x+y-w[p]$. An OLT has also to take into account the delays for the packets that were ungranted in the previous cycles of an ONU (ungranted time slot [p]). The transmission slot (seconds) for an ONU p is formulated at each cycle as (2) and the ungranted time slot for an ONU $\mathrm{p}$ is updated at each cycle as (3). Fig.1 (b) shows that, we achieve constant inter-window jitter by delaying the transmission of some packets (e.g. 4) to the next cycle.

$$
\begin{gathered}
w[p]=x+y-D_{E F}+\text { ungranted time slot }[p] \\
\text { ungranted time slot }[p]=\text { ungranted time slot }[p]+x-w[p]
\end{gathered}
$$

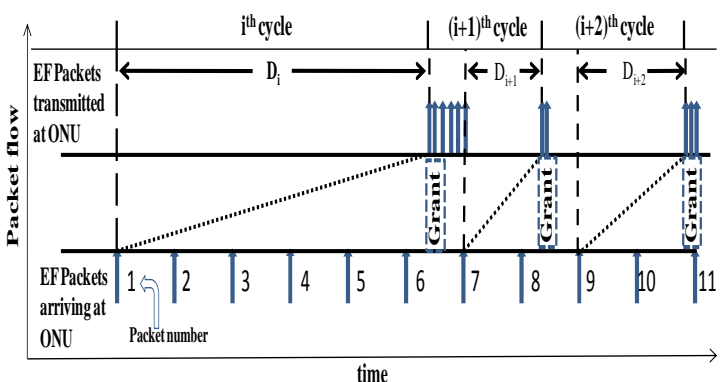

(a)

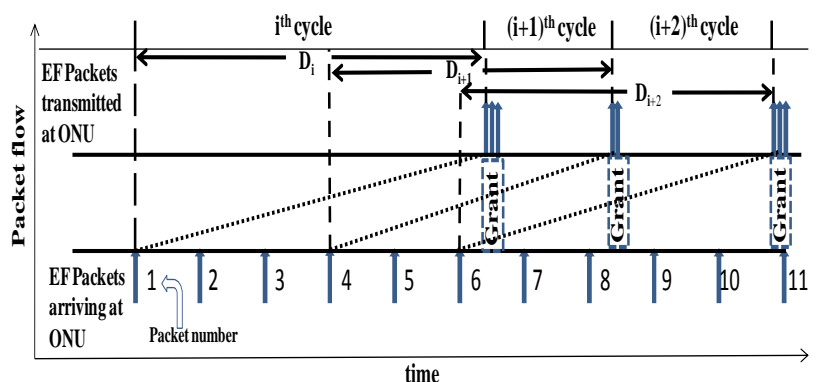

(b)

$\uparrow$ represents packets ...connects packet arrival and transmission represents time epoch of packets arrival and transmission of the first departed packet in the cycle

Figure 1: (a) Illustration of variation of the first departed EF packet delay due to variable cycle time in IPACT. (b) Illustration of constant first departed EF packet delay due to Delay-Aware Window Sizing (DAWS) approach.

The implementation of the DAWS approach is not without challenges. We see that the transmission slot for an ONU depends on the expected time duration of the next cycle. Since IPACT is an online approach, the time of issue of the grant message of the next cycle for an ONU may not be known at the time of the issue of the present grant message. Careful evaluation helps us to know that for an EPON comprising of $N$ ONUs, the time of issue of the $(i+1)^{\text {th }}$ grant to the $j^{\text {th }}$ ONU $\left(g t_{i+1}[j]\right)$ will depend on the $[i-1+\bmod (1, j)]$ report message of $[N-\bmod ((N-j+1), N)]$ ONU; where $\bmod (x, y)$ is the remainder of $(x / y)$. When the report messages from an ONU arrive, we determine the grant time of the next (in cyclic order) ONU. Using the latest determined grant time of an ONU $k$, we can calculate the maximum time epoch at which the $(i+1)^{\text {th }}$ grant to the $j^{\text {th }}$ ONU will be transmitted and is formulated by (4), where $\mathrm{T}_{\max }$ is the maximum transmission window per ONU and $r t t[p]$ is the round trip time of the $\mathrm{p}^{\text {th }}$ ONU.

$$
g t_{i+1}[j]=g t_{(i+\bmod (1, j))}[k]+r t t[k]-r t t[j]+(j-k+N *(1-\bmod (1, j))) *\left(T_{\max }\right)
$$

$D A G S$ (for AF traffic): The challenges that are to be met to provide constant delay performance to AF traffic are different. The AF traffic is bursty and thus the GBR methods are not applicable and thus we have to store all the report messages from ONUs. We translate the report message into newly requested bytes which depends on the present report, last report and the granted bytes in the last cycle. We store reports in a two array format. In one array we store the newly requested bytes and in the other one we assign the corresponding delay value. At each grant, we update the delay field and grant only those array values for which the delay exceeds a certain threshold value $\left(\mathrm{D}_{\mathrm{AF}}\right)$. Also, since AF traffic transmitted at the ONU will be different from traffic granted at the OLT (due to unused slot remainders [5]), the OLT has to account for the over (when the packet size is more than the transmission slot for AF traffic but less than the combined transmission slot for AF and BE traffic, the packet is transmitted leading to the over transmitted bytes) or under transmitted bytes (when the packet size is less than the combined transmission slot for $\mathrm{AF}$ and $\mathrm{BE}$ traffic, the packet is not transmitted leading to the under transmitted bytes) at ONU. The MAC protocol is suitably adapted to account for the reporting of the over or under transmitted bytes. 


\section{Simulation Results}

We study the performance of the proposed DBA scheme by conducting a simulation of an EPON access network with 16 ONUs. For our simulation study, we have assumed maximum ONU load of $100 \mathrm{Mbps}$, upstream bandwidth of the EPON as $1 \mathrm{Gbps}$, maximum OLT to ONU distance of $20 \mathrm{~km}$, maximum cycle time of $1.5 \mathrm{~ms}, \mathrm{D}_{\mathrm{EF}}=1.5 \mathrm{~ms}$, $\mathrm{D}_{\mathrm{AF}}=2 \mathrm{~ms}$ and guard time between adjacent slots as $1 \mu \mathrm{s}$. We generated traffic for different classes $(\mathrm{EF}, \mathrm{AF}$ and $\mathrm{BE}$ ) as in [2]. Fig.2 (a) gives the average delay of various traffic classes on the application of our algorithm. The average delay for EF traffic remains within the bound of $1.5 \mathrm{~ms}$ and the average delay for AF traffic remains constant at $2 \mathrm{~ms}\left(\mathrm{D}_{\mathrm{AF}}\right)$ for all loads. Fig.2 (b) shows that the inter-window jitter for DAWS approach at both half $(0.5)$ and full load (0.95) is centered at the same point whereas in traditional IPACT based approaches, it varies with load. The variance of the delay in the DAWS approach is improved at both loads. The variance $\left(\sigma^{2}\right)$ of the interwindow jitter for half load is $0.00215 \mathrm{~ms}^{2}$ and at full load is $0.00279 \mathrm{~ms}^{2}$ which shows that there is no variation in inter-window jitter with load. In Fig. 2 (c) we show that by adopting the DAGS approach, we are able to provide constant delay performance to even AF traffic with considerably reduced jitter. Fig.2 (d) shows that we have a much higher throughput of $95.5 \%$ compared to $83 \%$ in the HGP protocol and $82 \%$ in the CPBA-SLA protocol.

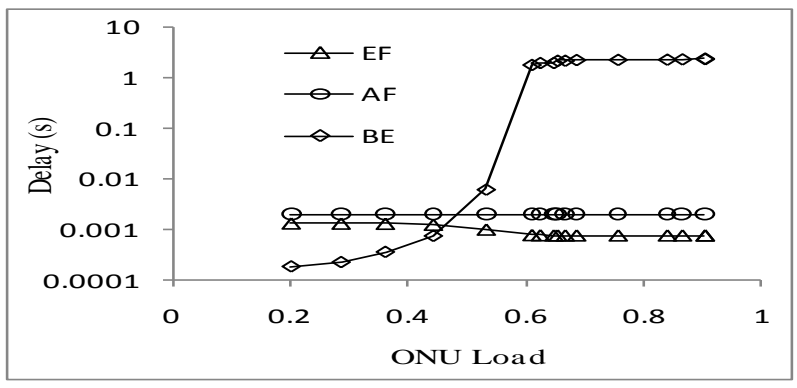

(a)

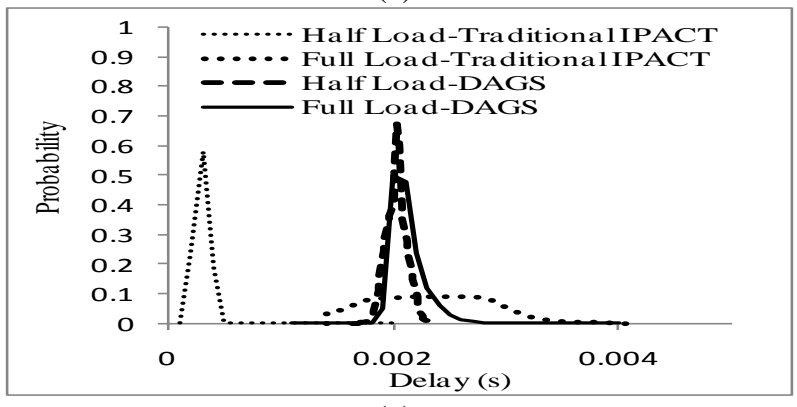

(c)

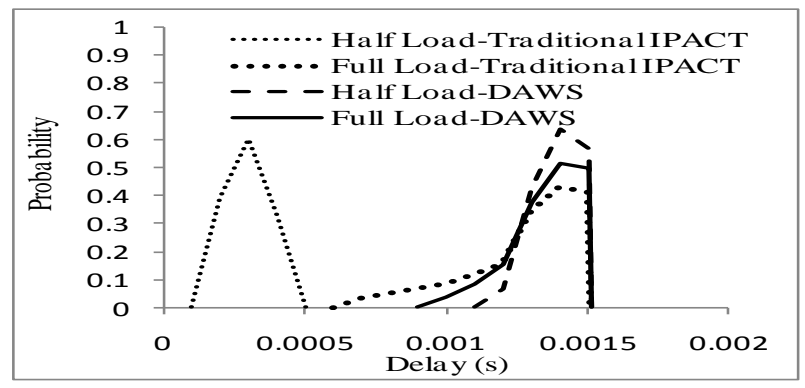

(b)

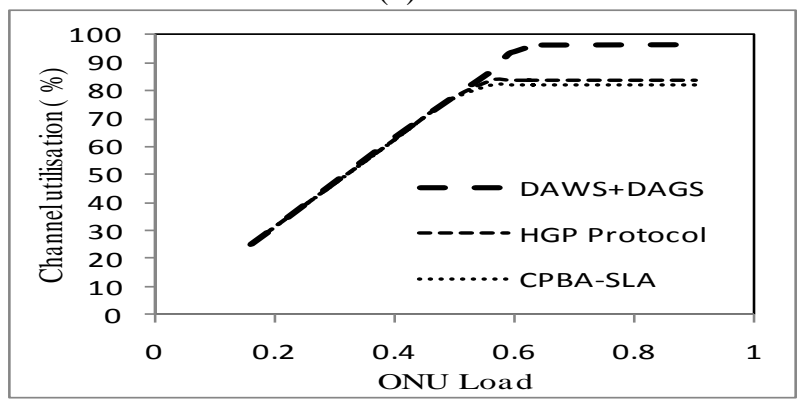

(d)

Figure 2: (a) shows the average delay for EF, AF and BE traffic on the application of our algorithm (b) shows the probability of the delay of the first departed EF packet for half and full load scenarios for traditional IPACT and the DAWS approach (c) shows the probability of the delay of the AF traffic for half and full load scenario for traditional IPACT and the DAGS approach (d) compares the channel utilization of proposed algorithm with the HGP and the CPBA-SLA protocol.

\section{Conclusion}

In this paper, we have proposed DAWS approach which minimizes inter-window jitter of EF traffic and maintains the delay within the desired bound. The proposed DAGS approach maintains the specified delay parameter for AF traffic and minimizes the jitter. All these gains are achieved at high throughput of about $95.5 \%$

\section{References}

[1] G. Kramer, B. Mukherjee, and G. Pesavento, "IPACT: A Dynamic Protocol for an Ethernet PON (EPON)," IEEE Communications Magazine, vol. 40, no. 2, pp. 74-80, February 2002.

[2] A.Shami, X. Bai, C. Assi, and N. Ghani, "Jitter performance in Ethernet passive optical networks," J. Lightwave Technol., vol. 23, no. 4, pp. 1745-1753, Apr. 2005.

[3] Su-il Choi and Jaehyung Park, "SLA-Aware Dynamic Bandwidth Allocation for QoS in EPONs", J. Optical Commun. Netw., vol. 2, no. 9, pp. 773-781, Sep. 2010

[4] F. An et al., "A new dynamic bandwidth allocation protocol with quality of service in ethernet-based passive optical networks," in Proc. International Conference on Wireless and Optical Communication (WOC 2003), Banff, Canada, Jul. 2003.

[5] G. Kramer, B. Mukherjee, and G. Pesavento, "Ethernet PON (ePON): design and analysis of an optical access network," Photon. Netw. Commun. 3(3), pp. 307-319, 2001. 